\title{
The Challenges of Collaborative Working: Bridging the Gap between Research and Practice
}

Steve W. Thompson ${ }^{1}$, David Rogerson ${ }^{1}$, Lee Bell ${ }^{1}$, David Hembrough ${ }^{1}$

${ }^{1}$ Academy for Sport and Physical Activity, Sheffield Hallam University, Sheffield, United Kingdom

\section{Introduction}

'Evidence-based practice', 'applied sport science' and 'bridging the gap': notions that frequent academic publications, underpin practice models and theme conference presentations. Conceptually similar, these terms both highlight and redress the estrangement between research and practice. No more apparent is this than within the field of Strength and Conditioning (S\&C), where practices and methods are often developed by practitioners within clubs and organisations out of necessity that are later investigated and published within academia.
Evidence-based practice was introduced within medicine in the early 90's, where research (high impact, peer-reviewed publications), clinical expertise (experience and knowledge of the patient) and the patient preferences (including the patient in the decision for courses of action) were triangulated as a means of working more systematically and holistically $(7,10,12)$. Attempts to translate this model into S\&C have been happening for some time (figure 1) with limited success, however. Despite their apparent simplicity in design and development, such approaches have proven to be difficult to implement in practice.

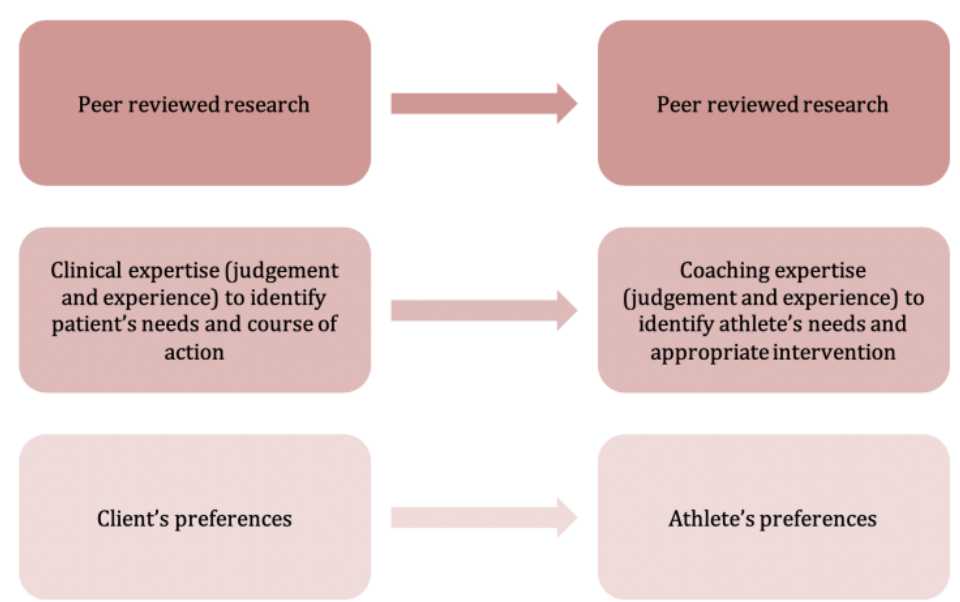

Figure 1. The translation of the evidence-based practice model from the medical field into the strength and conditioning field. 
The challenge of Performing EvidenceBased Research within an Applied

\section{Environment}

The fields of S\&C and sports science are ever-changing; they evolve at a precipitous pace; contain multiple, interconnected moving parts; and personnel shift rapidly and regularly within and throughout industry. Research, however, is comparatively stable; self-controlled with stoic traditions and time-honoured working practices that are adhered to rigidly to ensure robustness and scientific rigour. The integration of such dichotomous fields seems unnatural and counterintuitive. However, with a growing (and global) demand for coaches and practitioners, and a desire for more practically representative research, a harmonious and complementary existence between the two is both worthwhile and much needed.
Previously, Bishop (1) developed an Applied Research Model for the Sport Sciences (figure 2) with the aim of achieving the above. This model provides structure for researchers when developing research questions. It details a framework focussed on the development of sporting performance through a series of research projects, all aiming to build upon one another sequentially. Despite its scientific and systematic approach, this model still seems divorced from the realities of the applied world. For example, it is not until the eighth stage (potentially 3 - 4 studies deep) that the model suggests that research questions should be developed within the sporting setting itself. Therefore, given the lengthy research process (data collection, analysis, write-up and journal review - all aspects that can be time costly), it is possible that the need and timing for such questions has passed, rendering them obsolete whilst the field moves on.

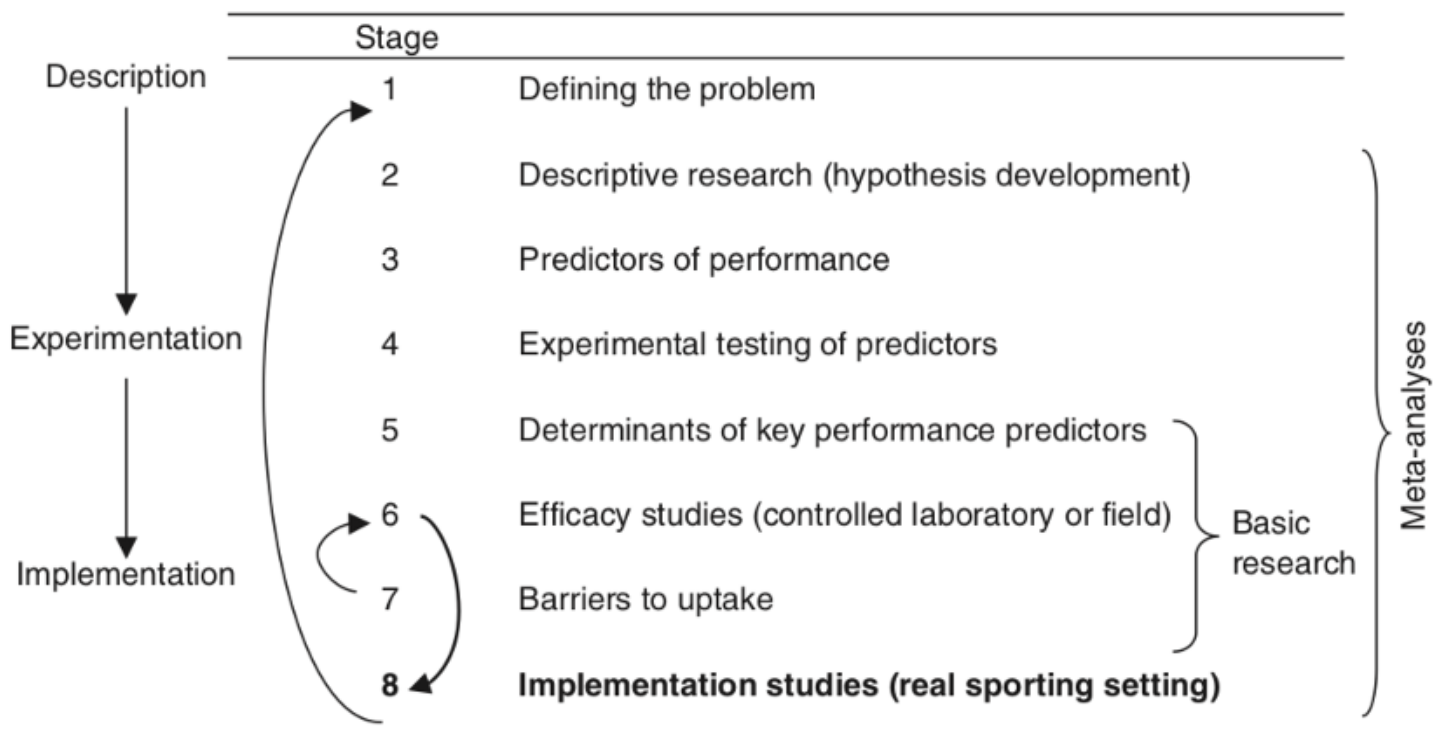

Figure 2. An applied research model for the sport sciences taken from Bishop (2008). The figure depicts the eight stages of the model and shows how it should be performed as a loop in order for future research questions to emerge. 
The traditional, quantitative research manuscript has specific requirements for it to be of adequate quality to be accepted for peer-reviewed publication. These include ethical approval and recruitment of large sample sizes; rigid and wellcontrolled methodologies; the use of advanced statistical procedures to model and evaluate data; the inclusion of control groups and the objective reporting of findings using the in-house writing style dictated by the journal of choice. Practitioners, therefore, can seem far removed from this method of communication. Strength and conditioning programmes within a 'real world', applied setting are often flexible and individualised, with intensities, volumes and exercises adapted on a sessional basis spanning the course of a season or competitive cycle and over a number of years as a form of long-term athlete development. This is in stark contrast with intervention or periodisation-based research studies, where typically participants follow a pre-planned training programme, often without deviation, for 6-12 weeks only $(13,14)$. It is also not uncommon for participants in such studies to be "recreationally active" or "collegiatelevel athletes" recruited from the home institution. The S\&C coach might therefore feel that such research is unrepresentative or difficult to replicate within an applied context, which necessitates pragmatism and adaptability to manage complexity and change. Research studies often evaluate the effect of a protocol or intervention through statistical procedures such as null hypothesis testing, by which the significance of a change in performance is measured by the production a $p$ value. This $p$ value, however - both in terms of its scope and magnitude - might leave practitioners asking, "what does this data actually mean?"
Journals such as the Journal of Strength and Conditioning Research (JSCR) and International Journal of Sports Physiology and Performance (IJSPP) require authors to include "Practical Applications" segments. These sections are there to translate the outcomes of the research into practice an outcome-orientated or "practice-based evidence" requirement of the publication. Nevertheless, these sections sometimes feel misplaced and disjointed, like add-on sections to the reader, with the practical relevance being an afterthought to a larger body of work. There is evidence to suggest that only $1.8 \%$ of practitioners acquire knowledge from journal articles (11). Further, Fullager et al. (4) observed that although $100 \%$ of coaches in a US sample ( $n=64$ ) utilised peer-reviewed journal articles, over $40 \%$ stated difficulties in implementing knowledge due to poor player compliance, staff buy-in, lack of staffing and/or time. Such issues can only be compounded by accessibility problems precipitated by the systems that govern research publication. Despite the obvious efforts by journals to improve their transference to a wider, non-academic sphere, researchers often find themselves not one, but two steps away from applied practice. As discussed, relinquishing the rigidity of well-controlled research designs is challenging enough to replicate that of applied practice (e.g. training and competition preparation), but the main role of a practitioner is to improve performance (e.g. matches, competitions or gold medals won). And, whilst research provides us with loose correlations between physical development and physical performance (e.g. developing maximal strength with the intention of increasing jumping or sprinting performance), translating these abilities to competitive performances in situ (e.g. scoring more baskets during a basketball 
competition) is even more difficult to decipher from rigid scientific research. Taking this into consideration, it is apparent that better ways of working and collaborating between researchers and practitioners must be presented in order to help to 'bridge this gap'.

\section{Moving Forward: Is there scope to "Bridge the Gap" between Research and Practice}

Although we have outlined some of the existing constraints of the successful integration of evidence-based practice, it is important to acknowledge that there are solutions available to both researcher and practitioner that will develop a much more harmonious and synchronous way of working. In a recent publication in IJSPP, Halperin (5) discussed the virtues of utilising case studies as a means of bridging the gap between researcher and practitioner. As discussed prior, and perhaps underpinned by the philosophical necessity to achieve external validity, or "generalisation" and eliminate biases, traditional quantitative research is often undertaken using group-based research

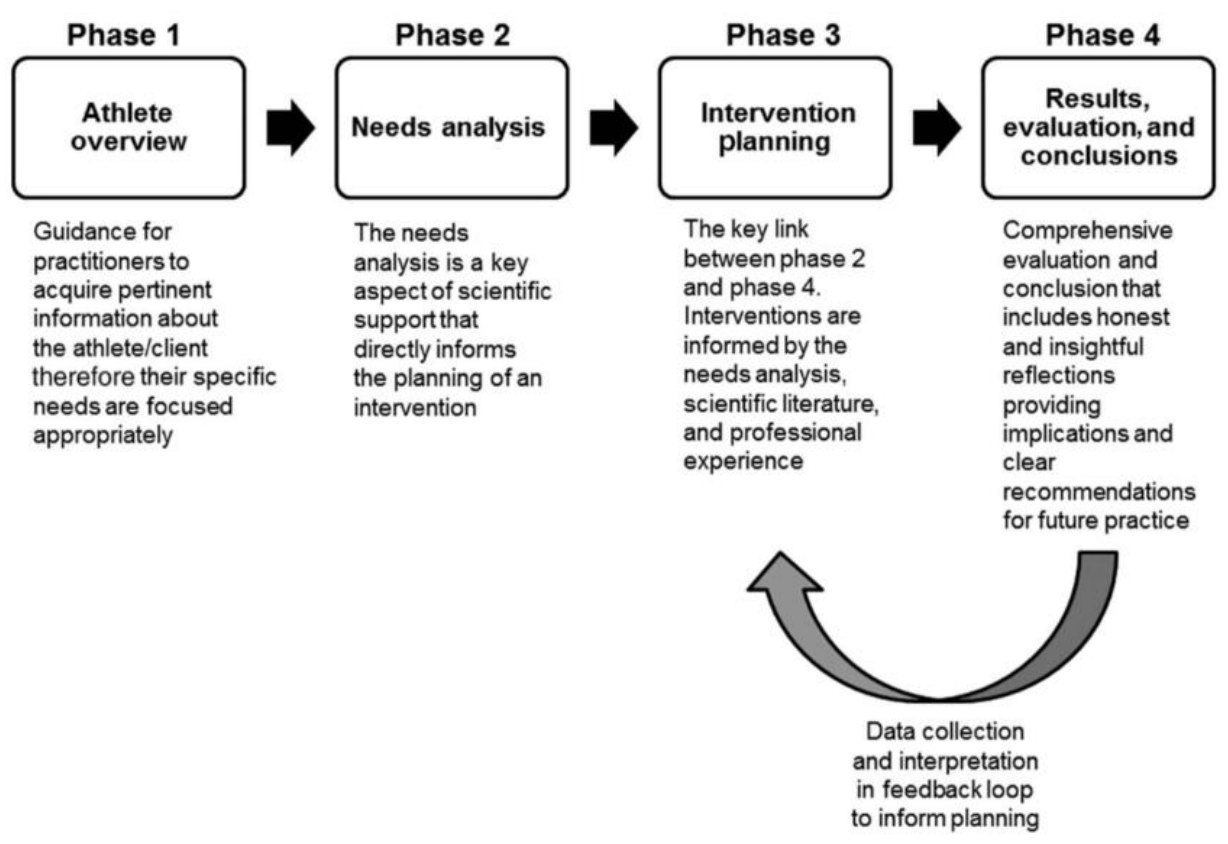

Figure 3. The scientific support model taken from Ruddock et al. (2019) designs; do not consider individual differences and are often rigid in their control and lack practical representativeness (9). Case studies, on the other hand, lend themselves to smaller sample sizes, encourage the reporting of a higher-level of participant information (that perhaps also account for individual differences) and allow for more complex designs such as longitudinal studies (5). Just as important, the development of case study-type research projects can foster new (or develop existing), working relationships between practitioners and researchers, bringing the practitioner into the research world and vice versa. Furthermore, all can be done with reduced interruption of every-day practices within the applied setting (often a criticism of traditional research) and still allow for the scientific rigour required for peerreviewed publication. Ruddock et al. (9) recently devised a working model for the development of case studies (figure 3), suggesting that with the abundance of scientific support that occurs with clubs, organisations and individual athletes, this type of research should be accessible. 
The inclusion of practitioners in the development of any research question should be at the forefront of research. Coaches will be asking questions daily but might not always link those questions to research. The fast-paced environment of applied sport creates an emotionally intelligent, reactive and reflective practitioner that can deliver effective and efficient programmes and procedures and generate large data sets over long periods of time (2). However, the reliability and validity of said data can often be lost due to a lack of time, knowledge, and desire. Thus, integrating the researcher into this environment can help to develop the structure, systems and scientific rigour needed to enable publication. The researcher can help to eliminate technological 'noise'; they can also help to ensure that procedures are reliable and that large datasets are analysed accurately and effectively (2) to facilitate publication and practice similarly. Whether this integration happens in-house through the employment of researchers within organisations, or working relationships are built with academic institutions and providers, this approach could help to bridge the gap we discuss, ensuring research is translatable, usable and representative of the applied world.

\section{Qualitative research: new insights}

Qualitative research is a common approach in disciplines such as psychology and exercise science, investigating human behaviour and the factors that affect it (8). The utilisation of this type of research in S\&C could further help to bridge the gap between science and practice. By interviewing, holding focus groups or producing questionnaires, researchers have a unique opportunity to gain 'insider knowledge' of the applied setting. Research models such as those by Bishop (1), Drust et al. (3) and Ruddock et al. (9) all begin with descriptive or 'defining the problem' type studies, which should utilise a coaches knowledge and experience as they are often the ones with 'the problem'. Uses for qualitative research could be, but not limited to, talent identification, translation of knowledge to practice or ethnography (6) and the evaluation of programmes. For example, a recent study by Stoszkowski and Collins (11) reported that from 320 coaches, $41 \%$ preferred to acquire knowledge through peer discussions with other coaches and practitioners. Knowing this information, therefore, can help to develop new and more accessible ways of working for researchers and individuals in the field.

\section{The role of the practitioner}

Whilst the suggestions so far have centred on the development of more accessible research, there are ways in which practitioners can utilise the knowledge already out there. The emergence and progression of popular podcasts (e.g. Pacey Performance, Sigma Nutrition Podcast, Guru Performance Podcast) open access publications and websites (e.g. Science for Sport), and subscription-based research reviews (e.g. Stronger by Science), platforms have been developed to disseminate research, either through specific topics of interest, or from researchers and practitioners themselves. Likewise, infographics broadcasted through social media (e.g. YLM Sports, Strength and Conditioning Research) provide information about the more outcome-focused elements of research that can be applied to practice without the constraints of reading full research papers. 
Technological advancements that facilitate peer-to-peer communication, online webinars and podcasts are also becoming increasingly popular. Increased access to face-to-face workshops and expert seminars provide a platform for researchers and coaches to present their work, philosophies and practices. Ensuring time for CPD type activities such as these can help practitioners acquire knowledge that can be utilised in their environments at the appropriate times. Similarly, through the production of more relaxed publishing platforms (e.g. International Universities Strength and Conditioning Association Journal and Sport Performance and Science Reports), less restricted research can be developed and accessed through a reader-review process as opposed to the more stringent peerreview process.

\section{Summary}

Evidence-based practice, bridging the gap and applied sport science in the current climate may still be challenging. Overcoming a number of barriers such as translation of research to applied practice, slow publishing processes and stringent requirements for traditional research, in addition to the fast-paced and multifaceted applied setting, options and solutions are available for researchers and practitioners to collaborate in a mutuallybeneficial and harmonious way and should be explored to suit the working environment. 


\section{References}

1. Laakso M, Bjork BC. Anatomy of open access Bishop D. An applied research model for the sport sciences. Sports Med 38: 253-263, 2008.

2. Coutts AJ. Working fast and working slow: the benefits of embedding research in high performance sport. Int $J$ Sports Phys Perf 11: 1-2, 2016.

3. Drust B, Green M. Science and football: evaluating the influence of science on performance. J Sports Sci 31: 1377-1382, 2013.

4. Fullager HHK, Harper LD, Govus A, McCunn R, Eissenmann J, McCall A. Practitioner perceptions of evidencedbased practice in elite sport in the United States of America. J Strength Cond Res 13: 2897-2904, 2019.

5. Halperin I. Case studies in sport and exercise sciences: a powerful tool to bridge the science-practice gap. Int J Sports Phys Perf 13: 824-825, 2018.

6. Harper LD, McCunn R. "Hand in glove": using qualitative methods to connect research to practice. Int J Sports Phys Perf 12: 990-993, 2017.

7. Jeffries I. Evidence-based practice is strength and conditioning: reality of fantasy? UKSCA J 39: 7-14, 2015.

8. Merriam SB, Tisdell EJ. Qualitative research: a guide to design and implementation. Hoboken, NJ: John Wiley \& Sons, 2015.

9. Ruddock AD, Boyd C, Winter EM, Ranchordas $M$. Considerations for the scientific support process and applications to case studies. Int J Sports Phys Perf 14: 134-138, 2019.

10. Sackett DL, Rosenberg WM, Gray JA, Haynes RB, Richardson WS. Evidence based medicine: what is it and what it isn't. Br Med J 312: 71-72, 1996.

11. Stoszkowski J, Collins D. Sources, topics and uses of knowledge by coaches. $J$ Sports Sci 34: 794-802, 2016.

12. Thomas A, Saroyan A, Dauphinee WD. Evidence-based practice: a review of theoretical assumptions and effectiveness of teaching and assessment interventions in health professions. Adv Health Sci Educ Theory Pract 16: 253-276, 2011.

13. Thompson SW, Rogerson D, Ruddock A, Barnes A. The effectiveness of two methods of prescribing load on maximal strength development: a systematic review. Sports Med 50: 919-938, 2020.

14. Williams TD, Tolusso DV, Fedwa MV, et al. Comparison of periodized and nonperiodized resistance training on maximal strength: a meta-analysis. Sports Med 47: 2083-2100, 2017. 